\title{
Circularization in B-type eclipsing binaries in the Magellanic Clouds ${ }^{\star}$
}

\author{
P. North ${ }^{1}$ and J.-P. Zahn ${ }^{2}$ \\ 1 Institut d'astronomie de l'Université de Lausanne, 1290 Chavannes-des-Bois, Switzerland \\ ${ }^{2}$ LUTH, Observatoire de Paris-Meudon, 92195 Meudon, France
}

Received 11 February 2003/ Accepted 21 March 2003

\begin{abstract}
Making use of detached eclipsing binaries discovered by the OGLE and MACHO teams in the SMC and in the LMC respectively, we give the value of the fractional radius above which circularization occurs. We show that this critical radius is consistent with that found for galactic binaries by Giuricin et al. (1984) and does not, therefore, depend strongly on metallicity. This conclusion is consistent with Zahn's (1975) theory of tidal dissipation. The critical radius seems slightly larger in the SMC than in the LMC, but this small difference is hardly significant and should be confirmed by a larger sample. We also explore empirically to what extent the critical radius might depend on stellar mass and surface gravity.
\end{abstract}

Key words. stars: eclipsing binaries - stars: fundamental parameters

\section{Introduction}

Synchronization between spin and orbital periods and orbital circularization are consequences of dissipative phenomena taking place in close binaries, generally designated as tidal effects. In stars with an outer radiation zone, which will be considered here, the main dissipative mechanism is radiative damping acting on the dynamical tide (Zahn 1975, 1977). In that case, the circularization timescale $t_{\text {circ }}=-\mathrm{d} t / \mathrm{d} \ln e$ is a very steep function of the fractional radius $R / a, R$ being the stellar radius, $a$ the semi-major axis of the relative orbit, and $e$ its eccentricity; indeed the theory predicts that $t_{\text {circ }} \propto(R / a)^{-21 / 2}$ (see Sect. 5).

Giuricin et al. (1984, hereafter GMM) have examined early-type (O, B, A-type) detached close binaries in our Galaxy. On the basis of a sample of about 200 binaries, they concluded that the transition between eccentric and circular or quasi-circular orbits (defined as having an eccentricity $e<$ $0.05)$ occurs at about $(R / a) \sim 0.24$, which agrees well with Zahn's theory.

In this paper, we have chosen to analyze the detached eclipsing binaries cataloged by Udalsky et al. (1998) in the SMC, and by Alcock et al. (1997) in the LMC. For these systems no radial velocity curve is available (except for a few recent exceptions), hence no spectroscopic orbit. However, they all have fairly good lightcurves, so that the product $e \cos \omega$ ( $e$ being the orbital eccentricity and $\omega$ the longitude of the

Send offprint requests to: $\mathrm{P}$. North,

e-mail: Pierre.North@obs.unige.ch

* This paper utilizes public domain data obtained by the OGLE Project described at http://sirius . astrouw. edu.pl/ ogle/ periastron) can be determined for each of them. In addition, all systems of a given Magellanic Cloud lie practically at the same distance, except for small depth effects which we consider negligible for our purpose. This allows one to have a good idea of the masses of the components, even though the exact distance is still a matter of debate.

In Sect. 2 we give more details on the definition of the sample and present the analysis of the lightcurves of the systems (part of which were made by other authors). The orbital elements are discussed in Sect. 3 in the context of circularization, and the possible dependence of the critical radius on gravity and mass are explored in Sect. 4. A short comparison with theory is presented in Sect. 5. Conclusions and hints for further work are given in Sect. 6.

\section{Lightcurve analysis}

\section{1. $S M C$}

All 153 detached systems listed by Udalski et al. (1998) in the SMC, and whose $I$ magnitudes are available on the OGLE web site ${ }^{1}$, have been analyzed using an interactive and graphical version of the EBOP16 code. The original version of this code was written by Etzel (1980), who kindly provided it to PN through the regretted Dr. Zdenek Kviz; it was modified by PN for better efficiency. This work was done before the publication of the two papers by Wyithe \& Wilson (2001, 2002) who systematically solved all OGLE lightcurves in the SMC cataloged by Udalski et al. (1998) but published only the

\footnotetext{
${ }^{1}$ http://sirius.astrouw.edu.pl/ ogle/
} 
parameters of some tens of systems which are most suitable for distance determination. In all cases, the ratio of radii $k$ was fixed to 1 , since it is well known that, except in the rare cases of complete eclipses, this parameter remains ill-defined, i.e. uncertain by $\pm 20 \%$ or more, especially when both components are similar (Andersen 1991; Andersen et al. 1980; North et al. 1997). The work of Wyithe \& Wilson (2001) on detached binaries confirms this, since their Fig. 3 shows hardly any correlation between real and fitted ratio of radii. It is worth noting that Alcock et al. (1997) also assumed $k=1.0$ in most cases for their binaries in the LMC. It is also well known that one of the most accurate parameters obtained from lightcurves of eclipsing binaries is the sum of radii of the components. Thus we obtain essentially the mean radius. The other fitted parameters are the orbital inclination $i$, the central surface brightness of the secondary $J_{\mathrm{S}}$ relative to that of the primary, and the products $e \cos \omega$ and $e \sin \omega$. The magnitude at quadrature and the exact epoch of the primary minimum were also adjusted. The linear limb-darkening coefficient was assumed equal to 0.18 , which is appropriate, in the $I$ passband, for main sequence stars with $T_{\text {eff }} \sim 15000-20000 \mathrm{~K}$, i.e. the vast majority of the sample members (van Hamme 1994). We used the lightcurves in the $I$ band only, because they are far more complete than those in the $B$ and $V$ bands. The typical precision of the fitted mean radius (formally the radius of the primary, but with a fixed ratio of the radii $\left.k=R_{\mathrm{s}} / R_{\mathrm{p}}=1.00\right)$ is $2-3 \%$.

In the meantime, Wyithe \& Wilson (2001) performed the same analysis, but using the more sophisticated WilsonDevinney code. They allowed the ratio of the radii to be adjusted but recognize that the result is generally not significant due to the degeneracy between the angle and this ratio $k$. Likewise, they stress the difficulty in recovering a reliable estimate of the eccentricity $e$. This is why we decided not to consider the eccentricity $e$ itself, but rather the product $e \cos \omega$, because the latter is determined in a much more reliable way from the lightcurve: it is directly related to the phase of the secondary minimum, which is very accurate and almost completely independent from any other parameter of the system.

In three cases, we had to revise the period given by Udalsky et al. (1998). This was done by PN using a period search code implementing the reciprocal of Renson's (1978) $\theta_{1}$ test, which was devised for $\alpha \mathrm{CVn}$ type variables but proved extremely useful for eclipsing binaries too. These three stars are listed in Table 1 with the new value of their period. Two of them have a well defined period while we can only list 3 possible periods to the third, which was not considered any more in the analysis.

\section{2. $L M C$}

The interpretation of the lightcurves was published by Alcock et al. (1997), using much the same assumptions as us. In particular, they assumed $k=1$ in $60 \%$ of cases. Although they did not classify their systems into the detached and non-detached categories, they classified them according to the morphology of their lightcurves. We have selected the systems having a type EA/EB 1 to 4, for which the depths of both minima are relatively similar, thus enhancing the probability that both
Table 1. Revised periods for three eclipsing binaries of Udalski et al. (1998).

\begin{tabular}{rcccl}
\hline \hline Area & Star No. & $\begin{array}{c}\text { spurious } \\
\text { period [d] }\end{array}$ & $\begin{array}{c}\text { correct } \\
\text { period [d] }\end{array}$ & Remark \\
\hline 4 & 192903 & 103.5902 & 102.8859 & well defined \\
9 & 134245 & 3.32492 & 9.97715 & \\
& & & 4.9855 & less good \\
& & & 2.4935 & less good \\
10 & 39758 & 3.35275 & 5.02945 & well defined \\
\hline
\end{tabular}

components have about the same mass and radius. Some semidetached and contact systems remain, but they will simply increase the relative number of circular orbits, which does not introduce any pertinent information. Interestingly, Alcock et al. also publish separately the $e \cos \omega$ and $e \sin \omega$ products, which allows us to use their results exactly in the same way as ours.

\section{Circularization and critical radius}

Instead of considering the classical eccentricity versus period diagram $(e-\log P)$, we choose to study $e \cos \omega$ as a function of relative average radius, since the latter is directly given by the lightcurves. The advantage of this diagram is that it contains a quantity directly pertinent in the theoretical predictions - the relative radius - while orbital period itself is less important. Figure 1a shows this diagram for all 152 detached binaries in the SMC. Qualitatively, it resembles closely the usual $e-\log P$ diagram: all eccentricities are allowed for small enough relative radii (long enough periods), and there is a critical value above which all orbits are circular. Figure $1 \mathrm{~b}$ shows an enlargement of this critical region.

Similarly, Fig. 2a shows all binaries of Alcock et al. (1997) considered for this study, while Fig. $2 b$ shows an enlargement of the critical region. Full dots refer to solutions of lightcurves obtained in the $V$ band, while open dots refer to lightcurves in the $R$ band. In the following, we adopt, for the LMC, results based on the $V$ lightcurves. Interestingly, Figs. 1 and 2 differ for $e \cos \omega$ values close to zero: while there is a smooth transition in the SMC, there is a gap, for the LMC, between null values and non-negligible ones. This is certainly due to the fact that Alcock et al. (1997) have put to zero very small eccentricity values when they considered them as non-significant; these authors fail to comment about this, so their exact criteria remain unknown, but this point is not crucial at all for our purpose.

Comparison of Figs. $1 \mathrm{~b}$ and $2 \mathrm{~b}$ suggests that the critical relative radius (above which all orbits are circular) is about $(R / a)_{\mathrm{c}}=0.25$ both for the SMC and for the LMC. Since the SMC is about two times more metal-poor than the LMC, this implies that metallicity plays no important role in the matter. These figures should also be compared with Fig. 2 of GMM giving $e$ versus $R / a$ for 200 early-type binaries in our Galaxy: interestingly, the critical radius is again the same, i.e. about 0.25 , confirming the absence of an important metallicity effect. 

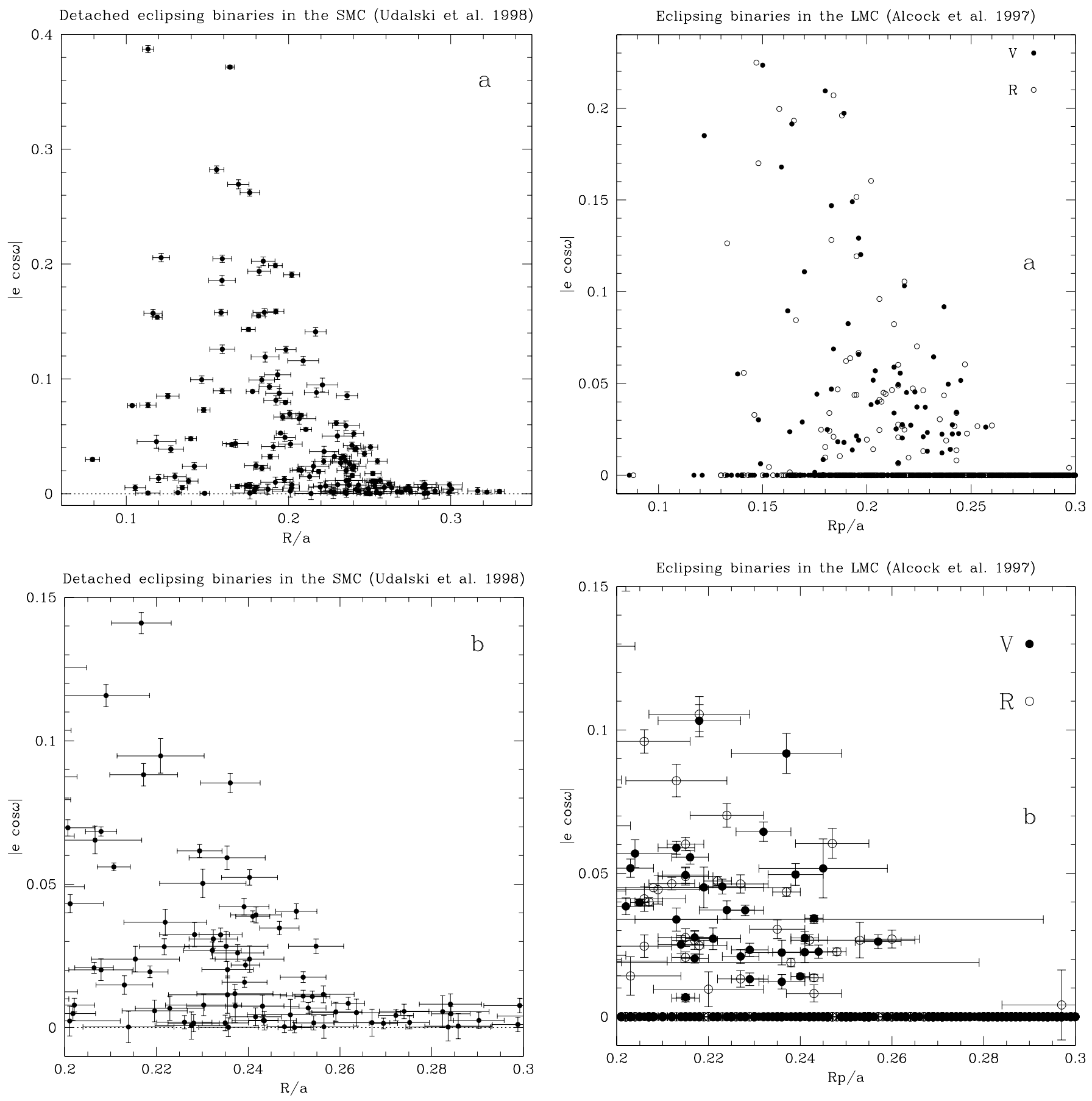

Fig. 1. a) $e \cos \omega$ versus relative radius for all detached eclipsing binaries in the SMC. b) Zoom of a) on the region around the critical radius.

Fig. 2. a) $e \cos \omega$ versus relative radius for all eclipsing binaries in the LMC listed by Alcock et al. (1997). Full dots: results based on the $V$ lightcurves. Open dots: results based on the $R$ lightcurves. b) Zoom of a) on the region around the critical radius.

An attempt at making a cleaner comparison between the SMC and LMC binaries was made, based on cumulative distributions of the relative critical radii. Four red giants were removed from the SMC sample. Then, for both the SMC and the LMC, all binaries for which $e \cos \omega<3 \sigma$, where $\sigma$ is the formal error provided by the EBOP (respectively the Wilson-Devinney) code, were dropped. The result is shown in Fig. 3. Both curves clearly converge toward $(R / a)_{\mathrm{c}} \sim 0.25$, although the slope is slightly smaller for the SMC than for the

LMC. The latter difference, which implies that there are more wider systems in the SMC, might be simply due to observational biases, e.g. time coverage, since the wider the system, the more difficult it is to detect narrow minima scattered in an otherwise constant curve.

The critical radius seems slightly larger in the case of the SMC $\left((R / a)_{\mathrm{c}} \sim 0.255\right)$ than in the case of the $\operatorname{LMC}\left((R / a)_{\mathrm{c}} \sim\right.$ $0.245)$ but the difference is so small that it seems hardly 


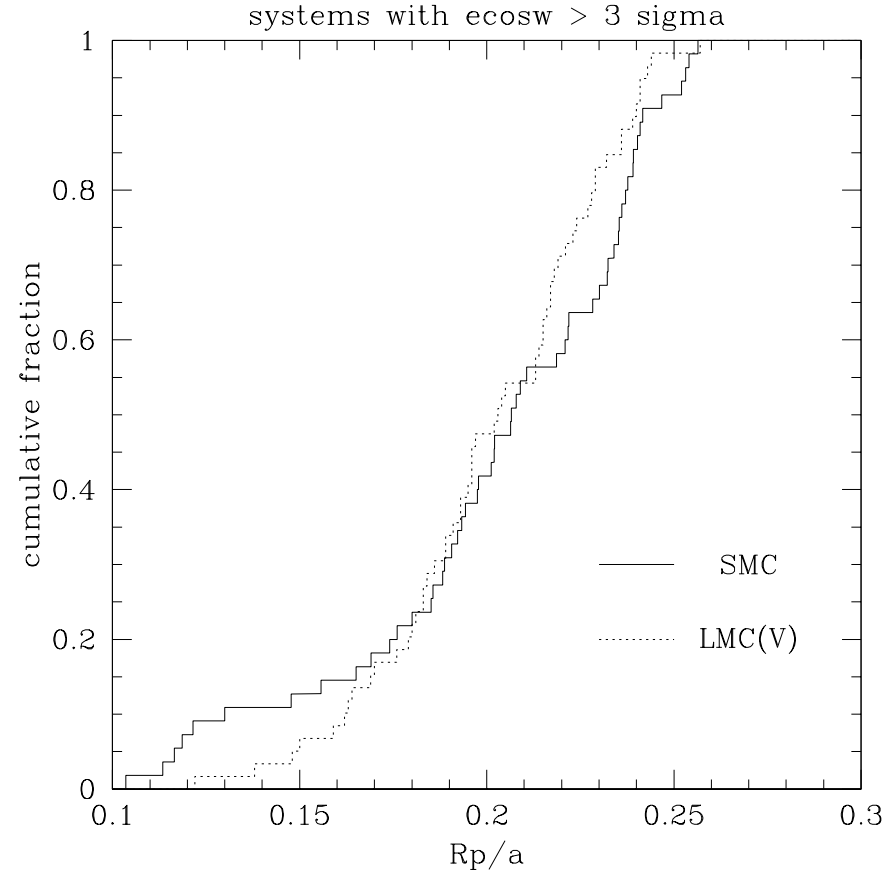

Fig. 3. Cumulative distribution of significant values of $e \cos \omega$ $(e \cos \omega>3 \sigma)$ for the SMC and LMC binaries.

significant. Therefore, our results suggest that the critical radius may be considered as a universal quantity.

\section{Does the critical radius depend on mass or gravity?}

\subsection{Mass determination}

In order to explore the possible effect of mass and surface gravity on critical radius, we have estimated the mass and surface gravity of all binaries which we judged appropriate for this purpose. Practically all binaries in the list of 153 detached systems in the SMC published by Udalski et al. (1998) are in this case, except for a few systems with evolved components, since both minima of their lightcurves present very similar depths. Generally, this is the sign that both components are similar as well. In the case of the LMC binaries, Alcock et al. (1997) classify the lightcurves according to the contrast between the depths of the secondary and primary minima, by a number varying from 1 (equal depths) to 9 (very shallow secondary minimum, typical of Algol-type binaries). We have selected the systems classified 1 to 4 in this regard, keeping only 354 stars (only 7 of which have classification 4) from the initial sample of 611 stars. It remains possible that the LMC sample be polluted by semi-detached or contact systems, while all systems considered here in the SMC are certainly bona fide detached ones.

The method of mass and gravity determination is the following:

- The distance to each Magellanic Cloud is assumed known. The "long" and "short" distance scales still have their respective advocates (e.g. Bono et al. 2001, 2002;
Udalski 2000), so we adopted some intermediate values: $(m-M)_{0}=18.9$ for the SMC, and $(m-M)_{0}=18.4$ for the LMC. So doing, we took into account the constraint that the difference of the distance moduli is 0.5 , with a rather good accuracy (Groenewegen 2000; Cioni et al. 2000) ${ }^{2}$. On the other hand, we neglected depth effects.

- The reddening was assumed constant on the whole field of each Cloud, the adopted mean values being $<E(B-V)>\quad=0.087$ and 0.143 for the SMC and the LMC respectively (Udalski 2000). Using the relation $A_{V}=3.1 \cdot E(B-V)$ (Cardelli et al. 1989), this gives $<A_{V}>=0.27$ for the SMC and 0.44 for the LMC.

- The absolute visual magnitude of the average component of each binary is computed through the formula $M_{V}=V-\left(m_{V}-M_{V}\right)_{0}-<A_{V}>+0.7526$, with $V=I+(V-I)$ in the case of the SMC (Udalski et al. 1998 do not give directly the $V$ magnitude in their Tables 2-12).

- The bolometric correction is computed according to the polynomial formula of Flower (1996), duly corrected according to Flower (1998). Although this correction is valid for stars with solar metallicity, the metallicity effect is expected to be small.

- On the basis of theoretical evolution models (Charbonnel et al. 1993 for $Z=0.004$; Schaerer et al. 1993 for $Z=$ 0.008 ), a first guess of the effective temperature is obtained through the relation $\log T_{\text {eff }}=4.275-0.0938 \cdot\left(M_{V}+0.5\right)$, assuming the star to be on the main sequence. Knowing $\log T_{\text {eff }}$ and $\log L$, one interpolates the mass in the theoretical evolutionary tracks.

- The first estimate of the mass is used to obtain the semimajor axis of the relative orbit through the 3rd Keplerian law, which is possible thanks to the knowledge of the period, which is very accurate.

- The relative radius being known from the photometric solution of the lightcurve, one can compute the absolute radius. The latter allows to make a new estimate of $\log T_{\text {eff }}$ through the current value of $\log L$.

- A new bolometric correction and a new $\log L$ are computed, leading to a new mass estimate. The process is iterated until $\Delta \log T_{\text {eff }}<0.002$.

This method converges well, but the precision on the mass is rather low, because in the theoretical $\mathrm{H}-\mathrm{R}$ diagram, lines of constant $M_{V}$ intersect lines of constant radius at a small angle. This stems from the rapid rise of $B C$ with increasing $T_{\text {eff }}$, as shown in Fig. 4. Thus, a small error on the absolute visual magnitude, on $A_{V}$ or on $B C$ propagates into a large error on mass: a realistic error of \pm 0.2 mag implies a relative error on mass of $\pm 20 \%$. But Fig. 4 also shows that lines of constant radius are almost orthogonal to evolutionary tracks, which shows that the evolutionary state, represented e.g. by $\log g$, is well constrained. The error on $\log g$ is minimized by the fact that, if mass is overestimated, then the radius is overestimated as well (though to a lesser extent), so that $g \propto M / R^{2}$ remains little changed.

\footnotetext{
${ }^{2}$ Eclipsing binaries can provide very accurate and direct distances, provided both radial velocity and light curves be determined (Paczynski 1997; Clausen 2000).
} 


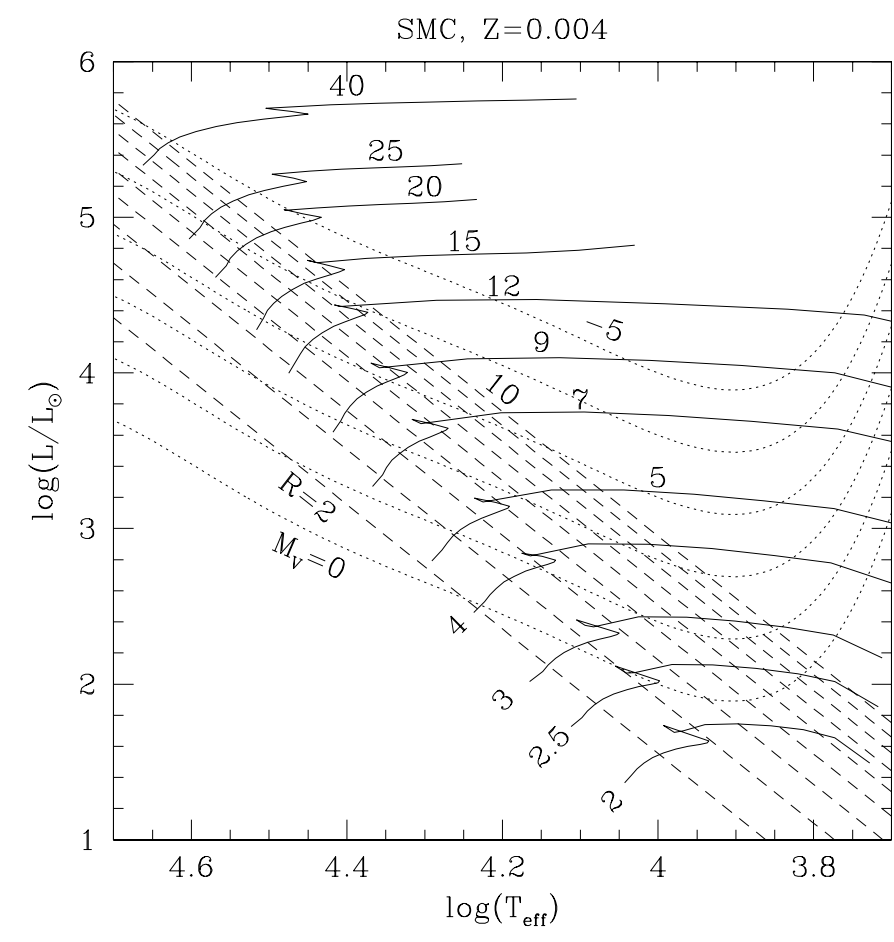

Fig. 4. Theoretical HR diagram for the SMC, assuming a metal content $Z=0.004$, a distance modulus $(m-M)_{0}=18.9$ and a visual absorption $A_{V}=0.27$. The continuous lines are the evolutionary tracks by Charbonnel et al. (1993), the broken lines are the loci of constant radius and the dotted lines are loci of constant absolute visual magnitude.

\subsection{Statistics with selection on mass or surface gravity}

The distributions of masses and $\log g$ of the SMC and LMC binary components obtained using the method just described are shown in Figs. 5 and 6 respectively. Although the distributions relative to the LMC are less reliable than those relative to the SMC because of probable pollution by semi-detached or contact systems, they are very similar. In addition, their shape is consistent with expectations: low mass stars are much more numerous than massive ones, in agreement with the IMF, and $\log g$ distribution is skewed toward large values because the early stages of the main sequence are longer lived than the late ones. In addition, the most frequent $\log g$ value is slightly larger in the SMC than in the LMC, which is consistent with the fact that metal-poor stars of a given mass have smaller radii than metal-rich ones (the SMC has $Z \sim 0.004$ while the LMC has $Z \sim 0.008)$.

\subsubsection{Gravity effects on the critical radius}

Let us focus on the possible gravity effect on the critical radius for circularization: discriminating stars by their $\log g$ value, one gets two cumulative distributions of the $R / a$ values (for significant $e \cos \omega$ ) for each Cloud, one for large gravities and the other for low gravities. The result is shown in Figs. 7a and 7b for the SMC and the LMC respectively.

Although the slope of the cumulative distribution of evolved stars is steeper than that of the unevolved stars, both
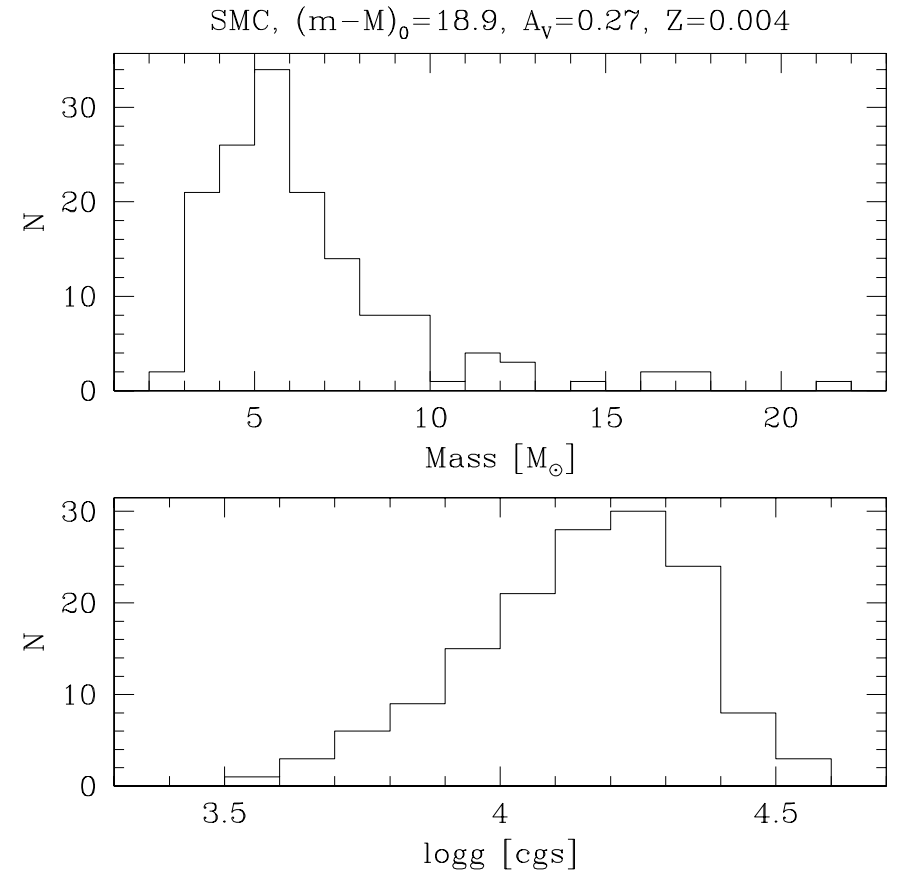

Fig. 5. Upper panel: mass distribution of detached eclipsing binary components (assuming both components equal) in the SMC. Lower panel: $\log g$ distribution of the same objects.
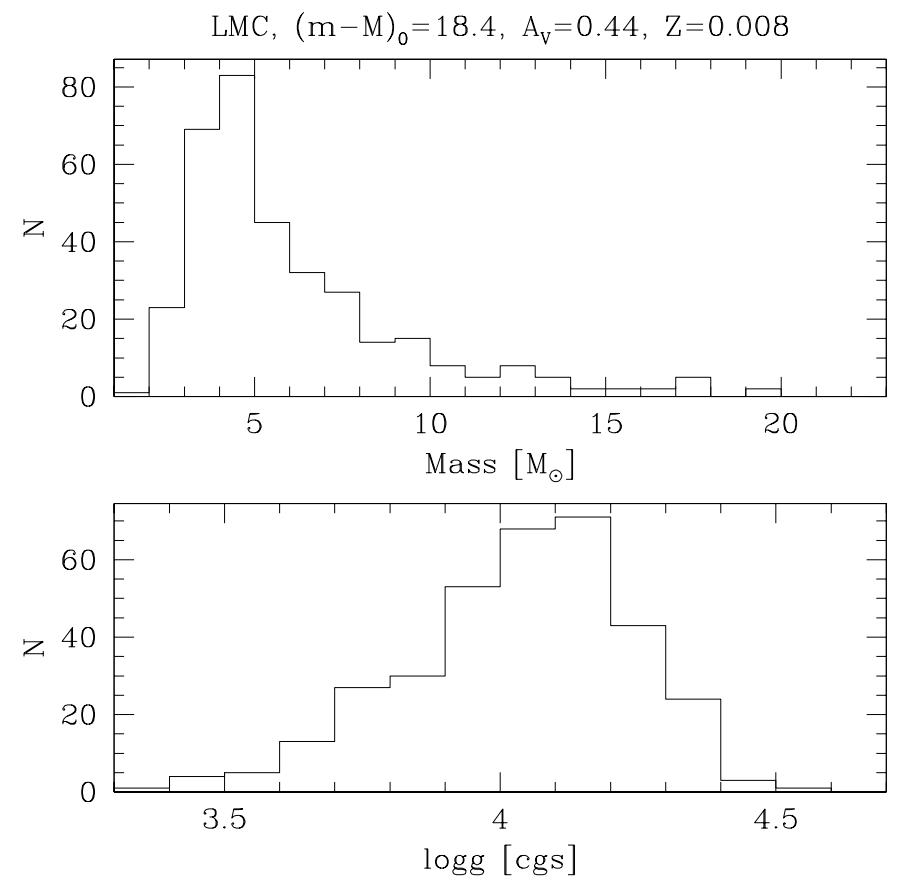

Fig. 6. Upper panel: mass distribution of detached eclipsing binary components (assuming both components equal) in the LMC. Lower panel: $\log g$ distribution of the same objects.

converge to the same value of $R / a$. The steeper slope simply indicates a larger number of components with radii close to the critical radius, which is expected from evolution of binaries with a homogeneous set of orbital parameters. 

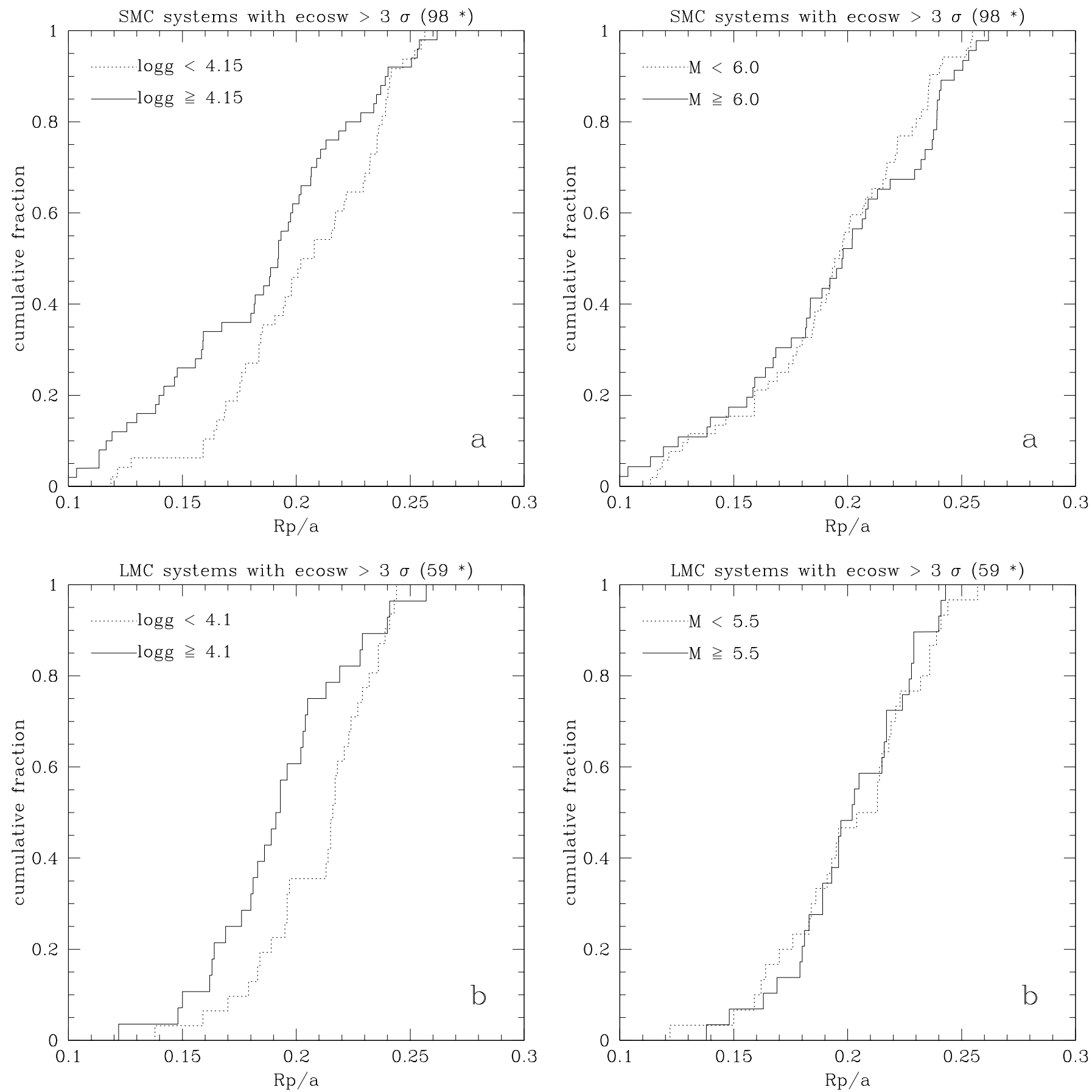

Fig. 7. a) Cumulative distribution of the relative radius corresponding to significant $e \cos \omega$, for main sequence detached eclipsing binaries in the SMC. The continuous line is for large gravity stars, the dotted line for low gravity ones. b) Same as a), but for the LMC.

\subsubsection{Mass effects on the critical radius}

Discriminating stars by their masses, we get the cumulative distributions of relative radii shown in Figs. $8 \mathrm{a}$ and $8 \mathrm{~b}$ for each of the Clouds.

It is difficult to see any significant trend as a function of mass: although one might suspect that, in the SMC, lower masses correspond to a lower critical radius, the reverse would hold in the LMC! In spite of the rather large number of binaries

Fig. 8. a) Cumulative distribution of the relative radius corresponding to significant $e \cos \omega$, for main sequence detached eclipsing binaries in the SMC. The continuous line is for massive stars, the dotted line for less massive ones. b) Same as a), but for the LMC.

used in this sample, the statistics remains insufficient to make any firm conclusion.

\section{Comparison with theory}

In binaries whose components possess outer radiation zones, orbital circularization is due mainly to radiative damping acting on the dynamical tide. The critical fractional radius for circularization depends on several parameters, but primarily on a 
structure constant $E_{2}$ and on the age $t_{\text {age }}$ of the star. The full expression is given by

$$
\begin{aligned}
\left(\frac{a}{R}\right)_{\mathrm{c}}^{21 / 2}= & 212^{11 / 6}\left(\ln \frac{e_{\mathrm{i}}}{e_{\mathrm{f}}}\right)^{-1}\left(\frac{G M}{R_{\mathrm{f}}^{3}}\right)^{1 / 2} \\
& \times \int\left(\frac{R}{R_{\mathrm{f}}}\right)^{9} E_{2} \mathrm{~d} t,
\end{aligned}
$$

with the usual notations, $e_{\mathrm{i}}$ and $e_{\mathrm{f}}$ being respectively the initial and final eccentricities and $R_{\mathrm{f}}$ the final radius (Zahn 1975, 1977). We have assumed that the binary components are of equal mass $M$, and that the rotation is synchronized with the orbital motion.

Let us apply this expression to a $15 M_{\odot}$ star whose age is half the main sequence lifetime $\left(t_{\mathrm{age}}=5.8 \times 10^{6} \mathrm{yrs}\right)$, and take $e_{\mathrm{i}} / e_{\mathrm{f}}=0.3 / 0.01$. For this purpose we may ignore the circularization which occurred during the pre-main sequence phase, since the envelope of the star was then completely radiative (Palla \& Stahler 1993); on the contrary, for solar-type stars, the PMS circularization can be more important than that on the MS (Zahn \& Bouchet 1989). We may also neglect the slight variation of $R$ and $E_{2}$ during MS evolution, and thus replace the integral above by $E_{2} t_{\text {age }}$. Under those conditions we get $(R / a)_{\mathrm{c}}=0.255$, in complete agreement with the observations presented above. This estimate is based on the models of Schaller et al. (1992) for a metallicity of $Z=0.020$, and the values of $E_{2}$ are taken from Claret \& Cunha (1997), who used more accurate models than in Zahn (1975).

Should we expect a variation of the critical radius with age, and therefore with the surface gravity? It is true that the radius increases by a factor of about 2 on the main sequence, but this increase occurs toward the end of that phase, where $E_{2}$ is dropping very fast by a factor of 1000 and more (cf. Claret \& Cunha), due to the shrinking of the convective core. Therefore the integral in (1) saturates at a level which is not much higher than its value at half MS lifetime, and hence there should be no detectable variation of $(R / a)_{\mathrm{c}}$ with gravity, as shown by the observations.

Since the structure constant $E_{2}$ is very sensitive to the size of the convective core (according to theory it scales as $\left.\left(R_{\text {conv }} / R\right)^{7}\right)$ in the limit of vanishing core), and since the latter increases as mass increases, one might expect that the critical radius $(R / a)_{\mathrm{c}}$ would slightly decrease accordingly. But the average age decreases too with mass, and so does the ratio $M / R^{3}$. If we take again $t_{\text {age }}$ as half the MS lifetime, the product $E_{2} t_{\text {age }}\left(M / R^{3}\right)^{1 / 2}$ turns out to be actually $16 \%$ smaller for a $15 M_{\odot}$ star than for a $5 M_{\odot}$ star, producing an increase of the critical radius of $1.5 \%$, which is not detectable. Thus the critical radius should not depend on mass, at least for stars of solar composition.

What about the role of metallicity? The convective core is slightly larger in lower metallicity stars, which means that $E_{2}$ is larger too. The structure constant has not been calculated yet for such stars, but one may estimate the effect by using the scaling of $E_{2}$ vs. $q_{\mathrm{c}}$, the fractional mass of the core, which may be deduced from the calculations by Claret \& Cunha: $E_{2} \propto q_{\mathrm{c}}^{5.5}$. The main sequence lifetimes are very close, but the radii at lower metallicity are $13 \%$ and $18 \%$ smaller, respectively for the 15 and $5 M_{\odot}$ star, where we have used the models by Charbonnel et al. (1993) for $Z=0.004$, relevant to the SMC. Taking all this into account, we find that the critical radius is about $5 \%$ smaller for the low metallicity star of $5 M_{\odot}$, whereas for the $15 M_{\odot}$ star, the decrease is only $3.5 \%$. This slight difference is too small to be detected. Furthermore, there should be no mass dependence of the critical radius in the SMC, nor in the LMC whose metallicity lies in between, which again is confirmed by the observational data.

\section{Conclusion}

Using B-type eclipsing binaries of both Magellanic Clouds, we have determined the critical relative radius $(R / a)_{\mathrm{c}}$, above which all orbits are circular. Its value is between 0.245 and 0.26 . This critical radius is nearly the same in the SMC and in the LMC, though there is a marginal indication for a smaller value in the LMC than in the SMC. No significant difference was found between $(R / a)_{\mathrm{c}}$ for stars near the ZAMS and those more evolved on the main sequence. Likewise, $(R / a)_{\mathrm{c}}$ seems to be the same for stars more massive than 5.5 or $6.0 M_{\odot}$ as for those less massive than this limit. A larger sample of binaries will be needed to drive stronger conclusions as to a possible different $(R / a)_{\mathrm{c}}$ value in the SMC than in the LMC. New data from e.g. OGLE-III may fulfill this need in the near future. In any case, it is worth mentioning that the $(R / a)_{\mathrm{c}}$ value found here in the Magellanic Clouds is quite consistent with that found by GMM for galactic binaries. This work is the first attempt at looking for the effect of metallicity, mass and surface gravity on the value of $(R / a)_{c}$.

These results are in excellent agreement with the theoretical predictions by Zahn $(1975,1977)$, which invoke as a tidal dissipation mechanism the radiative damping of the dynamical tide.

Acknowledgements. PN thanks Michel Mayor for a constructive remark, and Frédéric Pont for transmitting corrections to Table 6 of Flower (1996). This work has been partly supported by the Swiss National Science Foundation.

\section{References}

Alcock, C., Allsman, R. A., Alves, D., et al. 1997, AJ, 114, 326

Andersen, J. 1991, A\&ARv, 3, 91

Andersen, J., Clausen, J. V., \& Nordström, B. 1980, in Close binary stars: Observations and interpretation, IAU Symp. 88, ed. M. J. Plavec, D. M. Popper, \& R. K. Ulrich (Dordrecht: Reidel), 81

Bono, G., Caputo, F., \& Marconi, M. 2001, MNRAS, 325, 1353

Bono, G., Groenewegen, M. A. T., Marconi, M., \& Caputo, F. 2002, ApJ, 574, L33

Cardelli, J. A., Clayton, G. C., \& Mathis, J. S. 1989, ApJ, 345, 245

Charbonnel, C., Meynet, G., Maeder, A., Schaller, G., \& Schaerer, D. 1993, A\&AS, 101, 415

Cioni, M.-R. L., van der Marel, R. P., Loup, C., \& Habing, H. J. 2000, A\&A, 359, 601

Claret, A., \& Cunha, N. C. S. 1997, A\&A, 318, 187 
Clausen, J. V. 2000, in From extrasolar planets to cosmology: The VLT opening symposium, Proceedings of the ESO Symposium held at Antofagasta, Chile, 1-4 March 1999, ed. J. Bergeron, \& A. Renzini (Berlin Springer-Verlag), 225

ESA 1997, The HIPPARCos and Tycho Catalogues, ESA SP-1200

Etzel, P. B. 1980, EBOP user's guide (3rd edition), UCLA

Flower, P. J. 1996, ApJ, 469, 355

Flower, P. J. 1998, private communication to F. Pont (Geneva Observatory).

Giuricin, G., Mardirossian, F., \& Mezetti, M. 1984, A\&A, 134, 365

Groenewegen, M. A. T. 2000, A\&A, 363, 901

North, P., Studer, M., \& Künzli, M. 1997, A\&A, 324, 137

Paczynski, B. 1997, in The extragalactic distance scale, STScI Symp. Ser. 10, ed. M. Livio, M. Donahue, \& N. Panagia (Cambridge Univ. Press), 273
Palla, F., \& Stahler, S. 1993, ApJ, 418, 414

Renson, P. 1978, A\&A, 63, 125

Schaerer, D., Meynet, G., Maeder, A., \& Schaller, G. 1993, A\&AS, 98, 523

Schaller, G., Schaerer, D., Meynet, G., \& Maeder, A. 1992, A\&AS, 96, 269

Udalski, A., Soszyński, I., Szymański, M., et al. 1998, Acta Astron., 48,563

Udalski, A. 2000, Acta Astron., 50, 279

van Hamme, W. 1994, AJ, 106, 2096

Wyithe, J. S. B., \& Wilson, R. E. 2001, ApJ, 559, 260

Wyithe, J. S. B., \& Wilson, R. E. 2002, ApJ, 571, 293

Zahn, J.-P. 1975, A\&A, 41, 329

Zahn, J.-P. 1977, A\&A, 57, 383

Zahn, J.-P., \& Bouchet, L. 1989, A\&A, 223, 112 